\section{Cholesterol, lipoproteins and subclinical interstitial lung disease: the MESA study \\ Abstract}

We investigated associations of plasma lipoproteins with subclinical interstitial lung disease (ILD) by measuring high attenuation areas (HAA: lung voxels between -600 and -250 Hounsfield units) in 6700 adults and serum MMP-7 and SP-A in 1216 adults age 45-84 without clinical cardiovascular disease in Multi-Ethnic Study of Atherosclerosis. In cross-sectional analyses, each SD decrement in high density lipoprotein cholesterol (HDL-C) was associated with a 2.12\% HAA increment (95\% Cl $1.44 \%$ to $2.79 \%$ ), a $3.53 \%$ MMP-7 increment $(95 \% \mathrm{Cl} 0.93 \%$ to $6.07 \%)$ and a $6.37 \%$ SP-A increment $(95 \% \mathrm{Cl} 1.35 \%$ to $11.13 \%)$, independent of demographics, smoking and inflammatory biomarkers. These findings support a novel hypothesis that $\mathrm{HDL}-\mathrm{C}$ might influence subclinical lung injury and extracellular matrix remodelling.

Cardiovascular disease (CVD) is a prevalent comorbidity in adults with fibrotic interstitial lung disease (ILD), yet the mechanisms underlying this association remain unclear. ${ }^{1-3}$ Lipids and lipoproteins contribute to the pathogenesis of CVD and in recent years have been linked to a number of lung diseases, including asthma and COPD. ${ }^{45}$ However, few studies have looked at the role of lipids and lipoproteins in interstitial lung injury, inflammation and fibrosis. ${ }^{6} 7$

Imaging-based identification of lung injury, matrix remodelling and fibrosis in asymptomatic individuals is a novel way of studying subclinical ILD and may lead to a better understanding of the early causes of fibrosis. Two methods have been developed and validated to identify subclinical ILD: automated detection using quantitative CT densitometry to measure increased lung attenuation (high attenuation areas, HAA) and visual inspection for the presence of interstitial lung abnormalities (ILA). ${ }^{8-11}$ In the current study, we examined associations of plasma lipids and lipoproteins with HAA, ILA and serum biomarkers of lung inflammation and extracellular matrix remodelling (SP-A and MMP-7) in the Multi-Ethnic Study of Atherosclerosis (MESA). ${ }^{12-15} \mathrm{We}$ hypothesised that the presence of coronary artery calcium, high low density lipoprotein cholesterol (LDL-C), low high density lipoprotein cholesterol (HDL-C) and their respective components would be associated with HAA, ILA and higher MMP-7/SP-A levels independent of demographic characteristics, smoking and inflammatory biomarkers.

Full Methods are available in the online supplementary data.

The MESA is a multicentre, prospective cohort study of 6814 adults age 45-84 sponsored by the National Heart Lung and Blood Institute to investigate the progression of subclinical CVD. The participant selection criteria have been previously described. ${ }^{16}$ Notably, there were no selection criteria based on lung disease, respiratory symptoms or smoking history. MESA was approved by institutional review boards at all collaborating centres, and all consent for participation.

Lung attenuation was measured on cardiac CT scans performed at baseline. Quantitative image attenuation was measured using a modified version of the Pulmonary Analysis Software Suite at a single reading centre by trained readers. HAA was defined as the volume of imaged lung having CT attenuation values between -600 and -250 Hounsfield units. ${ }^{8} 9$ ILA was visually assessed on full lung CT scans, as previously described. ${ }^{9-11}$

Of the 6814 MESA participants, there were 6700 with available lipid measurements included in HAA analyses, 2391 in ILA analyses and 1216 in MMP-7 and SP-A analyses, with sampling frame previously described. ${ }^{9}$ The median (IQR) HAA was $4.2 \%(3.5 \%-5.4 \%)$ of total imaged lung.

In multivariable-adjusted models, there was a significant association between the presence of coronary artery calcium, HAA and ILA. The presence of coronary artery calcium was associated with $1.47 \%$ increment in HAA (95\% CI 0.19 to 2.77) and participants provided written informed

with a $57 \%$ greater odds of ILA (OR $1.58,95 \%$ CI 1.18 to 2.08$)$. Greater total cholesterol, HDL-C, LDL-C and triglyceride levels were each associated with lower HAA (table 1). However, the associations of both LDL-C and triglycerides with HAA were greatly attenuated by further adjustment for left ventricular function. On the other hand, the association between greater HDL-C and lower HAA was only marginally changed and remained significant (figure 1). This association also persisted across HDL particle size and for ApoA-1, the major protein component of HDL.

In a multivariable-adjusted model, there were no associations of baseline HDL-C, LDL-C, triglycerides, ApoA-1, ApoB, presence of diabetes mellitus, hypertension or statin use with the presence of ILA assessed at 10-year follow-up. In a multivariable-adjusted model (table 2), each SD decrement in HDL-C was associated with a 3.53\% increment in MMP-7 (95\% CI $0.93 \%$ to $6.07 \%$ ) and a $6.37 \%$ increment in SP-A $(95 \%$ CI $1.35 \%$ to $11.13 \%)$.

Additional analyses are presented in online supplementary tables S1-S9 and figures S1-S2.

In this large cohort of communitydwelling adults, the presence of coronary artery calcium was associated with two measures of subclinical ILD (HAA and ILA), providing further support for a link between CVD and ILD. ${ }^{1-3}$ We also found that lower plasma HDL-C and ApoA-1 were each associated with greater HAA and greater serum MMP-7 and SP-A levels, independent of smoking, demographic factors, inflammatory biomarkers and measures of left ventricular function, a finding consistent with a previous study

Table 1 Associations of cholesterol and lipoproteins with high attenuation area (HAA)

\begin{tabular}{llr}
\hline & \% change in HAA $(95 \% \mathrm{Cl})^{*}$ & p Value \\
\hline Total cholesterol & $-1.32(-1.89$ to -0.75$)$ & $<0.001$ \\
HDL-C, mg/dL & $-2.12(-2.79$ to -1.44$)$ & $<0.001$ \\
LDL-C, mg/dL & $-0.84(-1.41$ to -0.28$)$ & 0.004 \\
Triglycerides & $-0.82(-1.45$ to -0.18$)$ & 0.01 \\
Large HDL, 9.4-14 nm & $-1.10(-1.93$ to -0.26$)$ & 0.01 \\
Medium HDL, 8.2-9.4 nm & $-2.56(-3.31$ to -1.80$)$ & $<0.001$ \\
Small HDL, 7.3-8.2 nm & $-2.69(-3.44$ to -1.94$)$ & $<0.001$ \\
Large LDL, 20.5-23 nm & $-1.36(-2.06$ to -0.66$)$ & $<0.001$ \\
Small LDL, 18-20.5 nm & $-0.73(-1.56$ to 0.11$)$ & 0.09 \\
Apolipoprotein A-1 & $-1.66(-2.36$ to -0.97$)$ & $<0.001$ \\
Apolipoprotein B & $-0.69(-1.37$ to -0.01$)$ & 0.048
\end{tabular}

Solid lines separate distinct models. Each model includes all exposure variables listed and is additionally adjusted for age, gender, race/ethnicity, educational attainment, height, body mass index, waist circumference, smoking status, cigarette pack-years, glomerular filtration rate, diuretic use, statin use, presence of hypertension, presence of diabetes mellitus, coronary artery calcium, study site, milliampere dose, total volume imaged lung, per cent emphysema on CT, interleukin- 6 and $C$ reactive protein.

* Reported per SD in each exposure variable. 


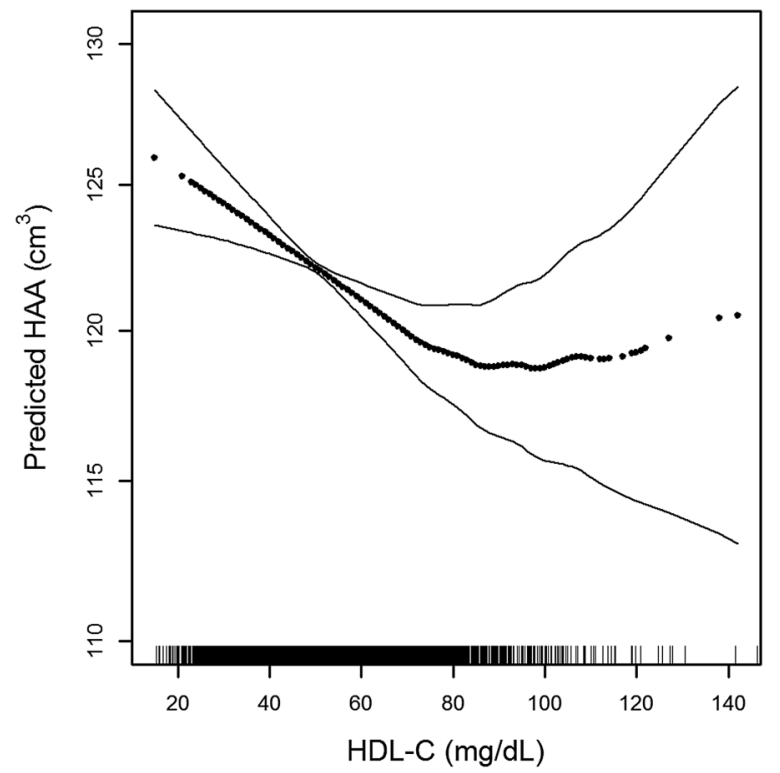

Figure 1 Continuous association between HDL-C and high attenuation areas (HAA) adjusted for age, gender, race/ethnicity, educational attainment, height, body mass index, waist circumference, smoking status, cigarette pack-years, presence of hypertension, presence of diabetes, low-density lipoprotein, triglycerides, C reactive protein, interleukin 6 , glomerular filtration rate, statin use, diuretic use, coronary artery calcium, study site, milliampere dose, total volume imaged lung, per cent emphysema on $\mathrm{CT}$, left ventricular ejection fraction and left ventricular end-diastolic mass; $p$ for non-linearity $0.009, p$ for association $<0.001$. Dark dotted line is the continuous association. Thin solid lines are the $95 \%$ confidence bands. Each point in the graph and each vertical hashmark in the rug plot along the $\mathrm{x}$ axis represent one study participant.

Table 2 Associations of cholesterol and lipoproteins with MMP-7 and SP-A

\begin{tabular}{lcccc} 
& $\begin{array}{l}\text { \% change in MMP-7 } \\
\text { (95\% Cl)* }\end{array}$ & p Value & $\begin{array}{l}\text { \% change in SP-A } \\
(95 \% \text { Cl)* }\end{array}$ & p Value \\
\hline Total cholesterol & $-0.01(-2.25$ to 2.28$)$ & 0.99 & $1.43(-2.95$ to 6.00$)$ & 0.52 \\
HDL-C, mg/dL & $-3.53(-6.07$ to -0.93$)$ & 0.008 & $-6.37(-11.13$ to -1.35$)$ & 0.01 \\
LDL-C, mg/dL & $0.35(-2.55$ to 1.89$)$ & 0.76 & $3.44(-0.98$ to 8.05$)$ & 0.13 \\
Triglycerides & $2.81(0.30$ to 5.37$)$ & 0.03 & $-3.11(-7.69$ to 1.70$)$ & 0.20 \\
Large HDL, 9.4-14 nm & $-4.10(-7.26$ to -0.84$)$ & 0.01 & $-14.39(-19.82$ to -8.59$)$ & $<0.001$ \\
Medium HDL, 8.2-9.4 nm & $-4.92(-7.61$ to -2.14$)$ & $<0.001$ & $-1.67(-7.06$ to 4.02$)$ & 0.56 \\
Small HDL, 7.3-8.2 nm & $-5.32(-8.03$ to -2.53$)$ & $<0.001$ & $-8.23(-13.31$ to -2.86$)$ & 0.003 \\
Large LDL, 20.5-23 nm & $-4.86(-7.50$ to -2.15$)$ & $<0.001$ & $-0.68(-6.02$ to 4.96$)$ & 0.81 \\
Small LDL, 18-20.5 nm & $-1.51(-4.73$ to 1.81$)$ & 0.37 & $-7.35(-13.18$ to -1.12$)$ & 0.02 \\
Apolipoprotein A-1 & $-2.83(-5.32$ to -0.27$)$ & 0.03 & $-4.79(-9.56$ to 0.12$)$ & 0.06 \\
Apolipoprotein B & $1.74(-0.64$ to 4.18) & 0.15 & $1.51(-3.06$ to 6.36$)$ & 0.52 \\
\hline
\end{tabular}

Solid lines separate distinct models. Each model includes all exposure variables listed and is additionally adjusted for age, gender, race/ethnicity, body mass index, smoking status, cigarette pack-years, presence of coronary artery calcium, statin use, interleukin 6 and $C$ reactive protein.

${ }^{*}$ Reported per SD in each exposure variable.

that found lower HDL-C levels in 39 adults with pulmonary fibrosis compared with healthy controls. ${ }^{6}$ Our findings suggest a novel hypothesis that HDL-C levels or its components might exert protective effects in subclinical ILD. Candidate mechanisms by which HDL-C might attenuate subclinical lung inflammation, extracellular matrix remodelling and fibrosis include modulation of endothelial function, protection against inflammation and oxidative stress, and alterations in surfactant function. ${ }^{17-19}$ ApoA-I has previously been shown to have beneficial effects in reducing lung inflammation and fibrosis in animal models, ${ }^{7}$ and greater small and medium-size HDL particle concentrations are associated with increased non-cardiovascular, non-cancer chronic inflammation-related deaths in MESA, ${ }^{20}$ supporting an anti-inflammatory role of small HDL particles in the lungs.

There are several limitations to our study, including the potential for residual confounding, the possibility that HAA likely includes pulmonary oedema, the lack of pathological validation of HAA and the use of cardiac rather than full lung CT scans to measure HAA. In addition, we found that HDL-C was not associated with ILA. This lack of consistency may reflect the 10-year latency period between baseline measurements of lipids and ILA, or it may reflect different stages of disease identified by the two measures of subclinical ILD. Furthermore, the clinical significance of elevated HAA, MMP-7 and SP-A in asymptomatic individuals is uncertain, limiting inferences about the role of HDL-C in fibrotic lung disease.

In summary, we found novel associations of greater HDL-C and ApoA-1 levels with lower HAA, a quantitative CT measure of subclinical lung inflammation and extracellular matrix remodelling, and with biomarkers of lung injury and extracellular matrix remodelling. This finding remains unexplained, but suggests a protective role of lipoproteins in ILD pathogenesis. More work is needed now to elucidate the mechanisms linking these two conditions, and the role that low lipoprotein levels might play in the pathogenesis of ILD.

Anna J Podolanczuk, ${ }^{1}$ Ganesh Raghu, Michael Y Tsai, ${ }^{3}$ Steven M Kawut, ${ }^{4}$ Eric Peterson, ${ }^{1}$ Rajiv Sonti, ${ }^{1}$ Daniel Rabinowitz, ${ }^{5}$ Craig Johnson, ${ }^{6} \mathrm{R}$ Graham Barr, ${ }^{1,7}$ Karen Hinckley Stukovsky, ${ }^{6}$ Eric A Hoffman, J Jeffrey Carr, ${ }^{9}$ Firas S Ahmed, ${ }^{10}$

David R Jacobs ${ }^{11}$ Karol Watson, ${ }^{12}$

Steven J Shea, ${ }^{1,7}$ David J Lederer ${ }^{1,7}$

${ }^{1}$ Department of Medicine, Columbia University Medical Center, New York, New York, USA

${ }^{2}$ Department of Medicine, University of Washington Seattle, Washington, USA

${ }^{3}$ Department of Laboratory Medicine and Pathology, University of Minnesota, Minneapolis, Minnesota, USA ${ }^{4}$ Department of Medicine, The Center for Clinical Epidemiology and Biostatistics, Perelman School of Medicine at the University of Pennsylvania,

Philadelphia, Pennsylvania, USA

${ }^{5}$ Department of Statistics, Columbia University, New York, New York, USA

${ }^{6}$ Department of Biostatistics, University of Washington, Seattle, Washington, USA

${ }^{7}$ Department of Epidemiology, Columbia University Medical Center, New York, New York, USA

${ }^{8}$ Departments of Radiology, Medicine, and Biomedical Engineering, University of lowa Carver College of Medicine, lowa City, lowa, USA

${ }^{9}$ Department of Radiology, Vanderbilt University Medical Center, Nashville, Tennessee, USA ${ }^{10}$ Department of Radiology, Columbia University Medical Center, New York, New York, USA ${ }^{11}$ Division of Epidemiology \& Community Health, University of Minnesota School of Public Health, Minneapolis Minnesota, USA

${ }^{12}$ Department of Medicine, David Geffen School of Medicine, University of California Los Angeles, Los Angeles, California, USA

Correspondence to Dr Anna Podolanczuk, Department of Medicine, Columbia University Medica 
Center, 622 W 168th St, PH-8, New York, NY 10032, USA; ajp2158@columbia.edu

Twitter Follow David Lederer @davidlederer

Acknowledgements The authors thank the other investigators, the staff and the participants of the Multi-Ethnic Study of Atherosclerosis (MESA) study for their valuable contributions. A full list of participating MESA investigators and institutions can be found at http://www.mesa-nhlbi.org.

Contributors AJP, DJL, RGB, SJS, KW and SMK conceived and designed the study. AJP, GR, MYT, RGB, EAH, FSA, SJS and DJL acquired the data. AJP, SMK, PLE, EP, RS, DR, CJ, RGB, KHS, JJC, DRJ, KW, SJS and DJL analysed the data. AJP drafted the initial manuscript. All authors contributed to the conception and design of the study and to the acquisition, analysis, or interpretation of data. All authors revised the manuscript for important intellectual content. All authors approved the final version of the manuscript. All authors agree to be accountable for all aspects of the work

Funding The work is funded by the National Institutes of Health contracts HHSN268201500003I, N01-HC-95159, N01-HC-95160, N01-HC-95161, N01-HC-95162, N01-HC-95163, N01-HC-95164, N01-HC-95165, N01-HC-95166, N01-HC-95167, N01-HC-95168 and N01-HC-95169 and grants UL1-TR-000040, UL1-TR-001079, R01-HL-103676, RC1-HL100543, R01-HL-093081, R01-HL-077612, T32-HL-105323 and K24-HL-131937; by the Pulmonary Fibrosis Foundation; and by the Rocco Guinta Research Fund.

Competing interests DJL has received modest consulting fees from Genentech/Roche, Boehringer Ingelheim, Gilead, Pharmakea, Veracyte, Patara Pharmaceuticals, Degge Group and the France Foundation related to IPF. Columbia University has received funding for clinical trials in IPF from Boehringer Ingelheim, Gilead, Bayer and Global Blood Therapeutics. Columbia University has received funding from the Pulmonary Fibrosis Foundation for DJL's consulting services. DJL has received modest fees for serving as a deputy editor for the Annals of the American Thoracic Society and as a statistical editor for Thorax. SMK reports grants from NIH during the conduct of the study and non-financial support from the ATS. He has received personal fees from the European Respiratory Journal for serving on an editorial board. The University of Pennsylvania has received grants from Actelion, United Therapeutics, Gilead, Lung Biotech and Bayer for CME courses. EAH is a founder and shareholder in VIDA Diagnostics, a company commercialising lung image analysis software developed, in part, at the University of lowa.

Ethics approval Institutional Review Boards at all collaborating centres.

Provenance and peer review Not commissioned; externally peer reviewed.

- Additional material is published online only. To view please visit the journal online (http://dx.doi.org/10. 1136/thoraxjnl-2016-209568).

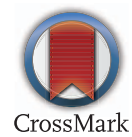

To cite Podolanczuk AJ, Raghu G, Tsai M Y, et al. Thorax 2017;72:472-474.

Received 11 October 2016

Revised 4 January 2017

Accepted 5 January 2017

Published Online First 27 January 2017

Thorax 2017:72:472-474

doi:10.1136/thoraxjnl-2016-209568

\section{REFERENCES}

1 Hubbard RB, Smith C, Le Jeune I, et al. The association between idiopathic pulmonary fibrosis and vascular disease: a population-based study. Am J Respir Crit Care Med 2008;178:1257-61.

2 Izbicki G, Ben-Dor I, Shitrit D, et al. The prevalence of coronary artery disease in end-stage pulmonary disease: is pulmonary fibrosis a risk factor? Respir Med 2009;103:1346-9.

3 Nathan SD, Basavaraj A, Reichner C, et al. Prevalence and impact of coronary artery disease in idiopathic pulmonary fibrosis. Respir Med 2010;104:1035-41.

4 Burkart KM, Manichaikul A, Wilk JB, et al. APOM and high-density lipoprotein cholesterol are associated with lung function and per cent emphysema. Eur Respir J 2014:43:1003-17.

5 Barochia AV, Kaler M, Cuento RA, et al. Serum apolipoprotein A-I and large high-density lipoprotein particles are positively correlated with FEV1 in atopic asthma. Am J Respir Crit Care Med 2015;191:990-1000.

6 Aihara K, Handa T, Nagai S, et al. Impaired endothelium-dependent vasodilator response in patients with pulmonary fibrosis. Respir Med 2013;107:269-75.

$7 \mathrm{Kim} \mathrm{TH}$, Lee $\mathrm{YH}$, Kim KH, et al. Role of lung apolipoprotein A-I in idiopathic pulmonary fibrosis: antiinflammatory and antifibrotic effect on experimental lung injury and fibrosis. Am J Respir Crit Care Med 2010;182:633-42.

8 Lederer DJ, Enright PL, Kawut SM, et al. Cigarette smoking is associated with subclinical parenchyma lung disease: the Multi-Ethnic Study of Atherosclerosis (MESA)-lung study. Am J Respir Crit Care Med 2009;180:407-14.

9 Podolanczuk AJ, Oelsner EC, Barr RG, et al. High attenuation areas on chest CT in community-dwelling adults: the MESA study. Eur Respir J

2016:48:1442-52.

10 Putman RK, Hatabu H, Araki T, et al. Association between interstitial lung abnormalities and all-cause mortality. JAMA 2016;315:672-81.

11 Washko GR, Hunninghake GM, Fernandez IE, et al. Lung volumes and emphysema in smokers with interstitial lung abnormalities. N Eng/ J Med 2011;364:897-906.

12 Greene KE, King TE Jr, Kuroki Y, et al. Serum surfactant proteins-A and -D as biomarkers in idiopathic pulmonary fibrosis. Eur Respir $J$ 2002:19:439-46.

13 Rosas 10, Richards TJ, Konishi K, et al. MMP1 and MMP7 as potential peripheral blood biomarkers in idiopathic pulmonary fibrosis. PLoS Med 2008, 5:e93.

14 Kinder BW, Brown KK, McCormack FX, et al. Serum surfactant protein-A is a strong predictor of early mortality in idiopathic pulmonary fibrosis. Chest 2009;135:1557-63.

15 Richards TJ, Kaminski N, Baribaud F, et al. Peripheral blood proteins predict mortality in idiopathic pulmonary fibrosis. Am J Respir Crit Care Med 2012;185:67-76.

16 Bild DE, Bluemke DA, Burke GL, et al. Multi-ethnic study of atherosclerosis: objectives and design. Am J Epidemiol 2002;156:871-81.

17 Pian MS, Dobbs LG. Lipoprotein-stimulated surfactant secretion in alveolar type II cells: mediation by heterotrimeric G proteins. Am J Physiol 1997; 273(Pt 1):L634-9.

18 Voyno-Yasenetskaya TA, Dobbs LG, Erickson SK et al. Low density lipoprotein- and high density lipoprotein-mediated signal transduction and exocytosis in alveolar type II cells. Proc Natl Acad Sci USA 1993:90:4256-60.

19 Tabet F, Vickers KC, Cuesta Torres LF, et al. HDL-transferred microRNA-223 regulates ICAM-1 expression in endothelial cells. Nat Commun 2014;5:3292.

20 Duprez DA, Otvos J, Tracy RP, et al. High-density lipoprotein subclasses and noncardiovascular, noncancer chronic inflammatory-related events versus cardiovascular events: the multi-ethnic study of atherosclerosis. J Am Heart Assoc 2015 4:e002295. 